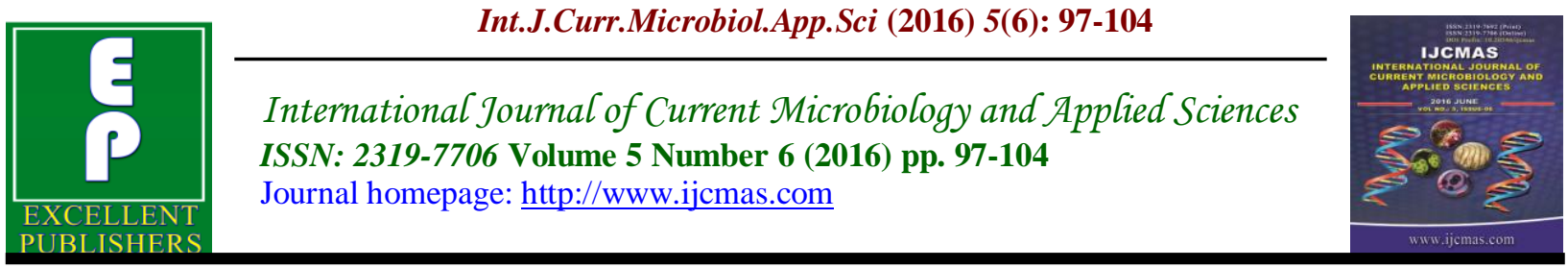

Original Research Article

http://dx.doi.org/10.20546/ijcmas.2016.506.012

\title{
Impact of Plant Density on the Sewage Treatment through selected Aquatic Macrophytes Using Angular Horizontal Subsurface Flow Constructed Wetland
}

\author{
V.P. Dhulap* and S.S. Patil \\ ${ }^{1}$ Department of Environmental Science, Solapur University, Solapur - 413255(MS), India \\ ${ }^{2}$ Department of Environmental Science, Dr. B.A.M. University, \\ Aurangabad- 431004, (MS), India \\ *Corresponding author
}

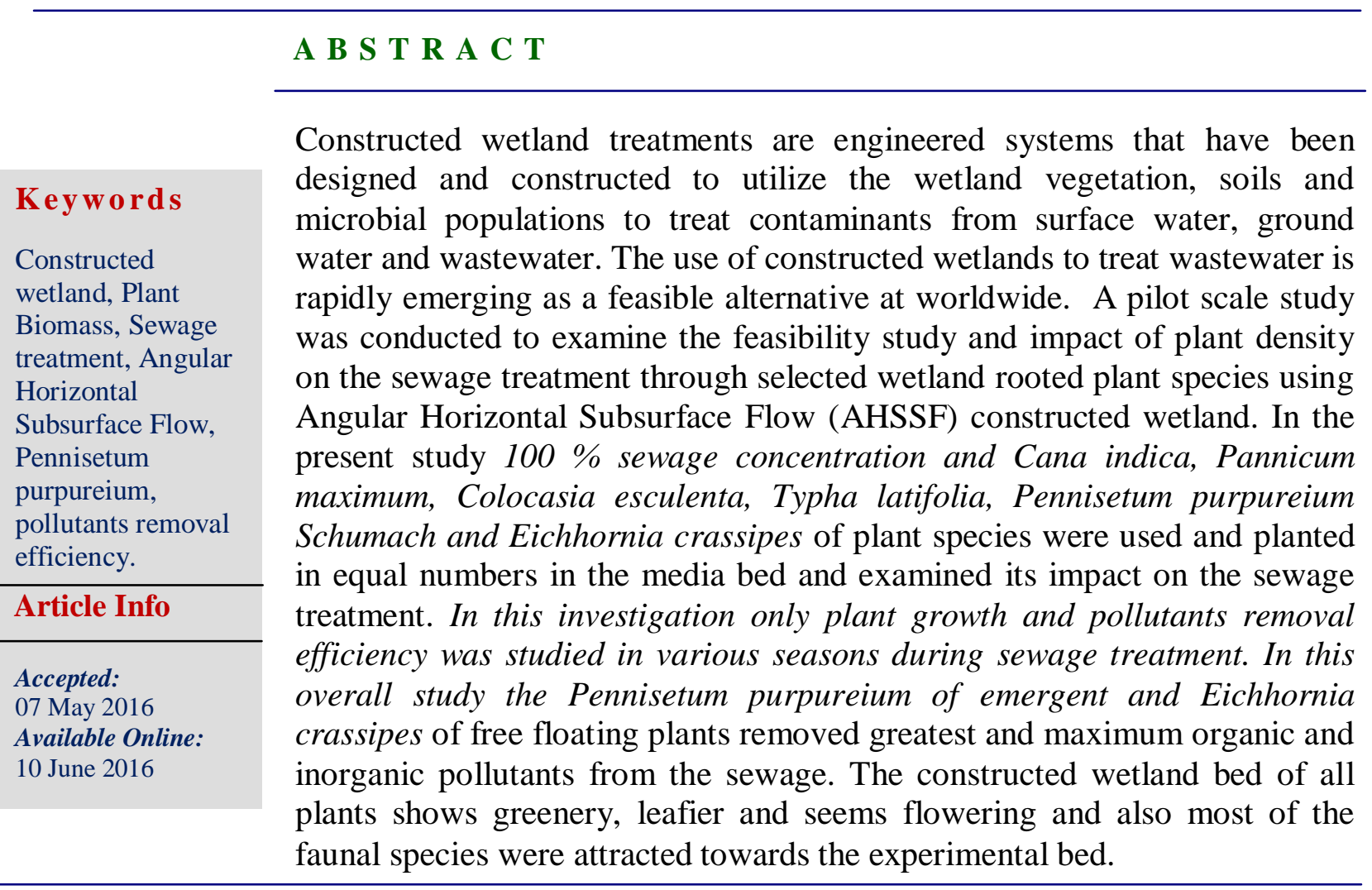

\section{Introduction}

The system of planting aquatic plants such as reeds or bulrushes in a wet (often gravel) substrate medium for gray water recycling is called a "Constructed Wetland" or "Artificial Wetland" or "Human Engineered Wetland". Constructed wetlands with emergent vegetation have been used to treat various types of wastewaters (Wallace and Knight, 2006). They are efficient in removal of organics through microbial degradation and settling of colloidal particles. Suspended solids are effectively removed via settling 
and filtration through the dense vegetation (Kadlec and Wallace, 2008). The use of constructed wetlands can be a cost-effective treatment alternative (Baltic Deal, 2011; Salomon and Sundberg, 2012; Hegazy et al., 2013). Subsurface flow wetland is considered to be advantageous as compared to the open surface wetlands and which are more commonly used for individual households. Constructed wetlands generally consist of one or more lined beds, or cells. Microorganisms and plants seem to work together symbiotically in constructed wetlands, as the population of microorganisms is much higher in the root areas of the plants than in the gravel alone. In comparison with conventional treatment systems, constructed wetlands have lower energy and chemical requirements. Another attraction of constructed wetlands is simplicity in operation, with less need for highly skilled manpower in day to day operations. They can be incorporated into the rural or urban landscape quite effectively, even in difficult terrain (Mayor and Sarana, 1950). Constructed wetlands are also appropriate for developing countries but they still have to become better known there (Mohamed, 2004; Heers, 2006; Kamau, 2009). Constructed wetlands have been used as an alternative option to treat wastewater.

The present study was conducted to assess the performance of AHSSF constructed wetland as a low cost facility to remediate (physically and biologically) pollution in wastewater phytoremediation treatment using planted macrophytes and study was conducted to examine the impact of plant density on the sewage treatment.

\section{Material and Methods}

In the present work planted equal numbers of plants in the plant media or in the media bed and this seems its impact on the sewage treatment. The experiment was performed in the Department of Environmental Science, Solapur University Solapur (M.S) India. In experimental designing and fabrication of constructed wetlands there are three sets of buckets with different sizes and dimensions were used. The root zone bed set was prepared in three layers which were prepared with pebbles, sand and garden sieved soil. Selective healthy, small, young, locally available saplings of macrophytes were transplanted and which were arranged in rows and columns. The plant bed was provided $10^{0}$ slopes and kept in the slanting position. Inlet and outlet flow rates were preset by using bucket and timer (Dhulap and Patil, 2014; Dhulap et al., 2014). In this study $100 \%$ sewage concentration were tested using Cana indica, Pannicum maximum, Colocasia esculenta, Typha latifolia, Pennisetum purpureium Schumach and Eichhornia crassipes plant species. Each plant species of their densities and their treatment efficiencies were calculated.

\section{Result and Discussion}

Cana indica plant growth results shows that, plant biomass or number of Cana was increase 40 numbers at 0 day, 42 at $1^{\text {st }}$ day, 45 at $2^{\text {nd }}$ day, 48 at $3^{\text {rd }}$ day, 53 at $4^{\text {th }}$ day and 59 numbers at $5^{\text {th }}$ day respectively (Fig. 1). On the same time studied sewage characteristics at 0 day to $5^{\text {th }}$ day. The sewage result reveals $\mathrm{pH}$ values were changed by 6.83 at 0 day to 7.3 at $5^{\text {th }}$ day. BOD $(\mathrm{mg} / \mathrm{L})$ was reduced by 51.44 at 0 day to 3.44 at $4^{\text {th }}$ day. COD $(\mathrm{mg} / \mathrm{L})$ was reduced by 118 at 0 day to 8.9 at $4^{\text {th }}$ day. Study showed the maximum pollutants removed at $4^{\text {th }}$ day (Table 1$)$.

Pannicum maximum shows that, plant biomass or number of Pannicum was increase 40 numbers at 0 day, 48 at $1^{\text {st }}$ day, 66 at $2^{\text {nd }}$ day, 74 at $3^{\text {rd }}$ day, 79 at $4^{\text {th }}$ day and 
88 numbers at $5^{\text {th }}$ day respectively (Fig. 2). The $\mathrm{pH}$ values were changed by 6.79 at 0 day to 7.29 at $5^{\text {th }}$ day. BOD $(\mathrm{mg} / \mathrm{L})$ was reduced by 42.0 at 0 day to 3.50 at $4^{\text {th }}$ day. $\mathrm{COD}(\mathrm{mg} / \mathrm{L})$ was reduced by 118 at 0 day to 11.2 at $4^{\text {th }}$ day. Study showed the maximum pollutants removed at $4^{\text {th }}$ day (Table 2 ).

Colocasia esculenta shows that, plant biomass or number of Colocasia was increase 40 numbers at 0 day, 41 at $1^{\text {st }}$ day, 46 at $2^{\text {nd }}$ day, 54 at $3^{\text {rd }}$ day, 63 at $4^{\text {th }}$ day and 69 numbers at $5^{\text {th }}$ day respectively (Fig. 3). The $\mathrm{pH}$ values were changed by 6.79 at 0 day to 7.91 at $5^{\text {th }}$ day. BOD $(\mathrm{mg} / \mathrm{L})$ was reduced by 42.0 at 0 day to 3.82 at $4^{\text {th }}$ day. $\mathrm{COD}(\mathrm{mg} / \mathrm{L})$ was reduced by 121 at 0 day to 11.86 at $4^{\text {th }}$ day respectively. Study showed the maximum pollutants removed at $4^{\text {th }}$ day (Table 3).

Table.1 Effect of plant growth of Cana indica (Density) on wastewater treatment through various parameters at $100 \%$ concentration of sewage

\begin{tabular}{|l|l|l|l|l|l|l|l|}
\hline S.N. & Parameters & 0 Day & $\mathbf{1}^{\text {st }}$ Day & $\mathbf{2}^{\text {nd }}$ Day & $\mathbf{3}^{\text {rd }}$ Day & $\mathbf{4}^{\text {th }}$ Day & $\mathbf{5}^{\text {th }}$ Day \\
\hline 1. & $\begin{array}{l}\text { Plant Growth } \\
(\text { Numbers })\end{array}$ & 40 & 45 & 48 & 53 & 59 \\
\hline 2. & $\mathrm{Ph}$ & 6.83 & 7.8 & 7.65 & 7.56 & 7.43 & 7.03 \\
\hline 3. & $\mathrm{EC}(\mu \mathrm{Moh} / \mathrm{Cm})$ & 2.56 & 1.12 & 0.92 & 0.88 & 0.82 & 0.84 \\
\hline 4. & $\mathrm{TSS}(\mathrm{mg} / \mathrm{L})$ & 394 & 223 & 203 & 109 & 100 & 102 \\
\hline 5. & $\mathrm{TDS}(\mathrm{mg} / \mathrm{L})$ & 1364 & 1129 & 961 & 612 & 458 & 462 \\
\hline 6. & $\mathrm{TS}(\mathrm{mg} / \mathrm{L})$ & 1758 & 1352 & 1164 & 721 & 558 & 564 \\
\hline 7. & $\mathrm{BOD}(\mathrm{mg} / \mathrm{L})$ & 51.44 & 37.04 & 12.36 & 7.27 & 3.44 & 3.48 \\
\hline 8. & $\mathrm{COD}(\mathrm{mg} / \mathrm{L})$ & 118 & 92.82 & 31.08 & 19.02 & 8.9 & 9.1 \\
\hline 9. & $\mathrm{NO}_{3}(\mathrm{mg} / \mathrm{L})$ & 26.8 & 19.06 & 15.02 & 9.05 & 3.02 & 3.06 \\
\hline 10. & $\mathrm{PO}_{4}(\mathrm{mg} / \mathrm{L})$ & 21.60 & 17.81 & 12.48 & 7.08 & 2.41 & 2.48 \\
\hline 11. & $\mathrm{SO}_{4}(\mathrm{mg} / \mathrm{L})$ & 107 & 94.0 & 78.0 & 31.0 & 19.46 & 19.51 \\
\hline
\end{tabular}

Table.2 Effect of plant growth of Panicum maximum (Density) on wastewater treatment through various parameters at $100 \%$ concentration of sewage

\begin{tabular}{|l|l|l|l|l|l|l|l|}
\hline S.N. & Parameters & 0 Day & $\mathbf{1}^{\text {st }}$ Day & $\mathbf{2}^{\text {nd }}$ Day & $\mathbf{3}^{\text {rd }}$ Day & $\mathbf{4}^{\text {th }}$ Day & $\mathbf{5}^{\text {th }}$ Day \\
\hline 1. & $\begin{array}{l}\text { Plant Growth } \\
(\text { Numbers })\end{array}$ & 40 & 48 & 66 & 74 & 79 & 88 \\
\hline 2. & $\mathrm{pH}$ & 6.79 & 6.96 & 7.86 & 7.52 & 7.21 & 7.29 \\
\hline 3. & $\mathrm{EC}(\mu \mathrm{Moh} / \mathrm{Cm})$ & 2.63 & 2.12 & 1.89 & 1.07 & 0.89 & 0.93 \\
\hline 4. & $\mathrm{TSS}(\mathrm{mg} / \mathrm{L})$ & 392 & 291 & 143 & 112 & 97 & 102 \\
\hline 5. & TDS $(\mathrm{mg} / \mathrm{L})$ & 1360 & 1091 & 784 & 490 & 465 & 469 \\
\hline 6. & $\mathrm{TS}(\mathrm{mg} / \mathrm{L})$ & 1752 & 1382 & 927 & 602 & 562 & 571 \\
\hline 7. & $\mathrm{BOD}(\mathrm{mg} / \mathrm{L})$ & 42.0 & 34.8 & 21.6 & 9.26 & 3.50 & 3.54 \\
\hline 8. & $\mathrm{COD}(\mathrm{mg} / \mathrm{L})$ & 118 & 92.7 & 36.7 & 21.8 & 11.2 & 11.7 \\
\hline 9. & $\mathrm{NO}_{3}(\mathrm{mg} / \mathrm{L})$ & 26.8 & 19.6 & 11.7 & 9.3 & 3.9 & 4.2 \\
\hline 10. & $\mathrm{PO}_{4}(\mathrm{mg} / \mathrm{L})$ & 21.60 & 17.92 & 12.41 & 8.66 & 2.80 & 3.12 \\
\hline 11. & $\mathrm{SO}_{4}(\mathrm{mg} / \mathrm{L})$ & 107.0 & 89.0 & 66.0 & 39.0 & 22.0 & 24.7 \\
\hline
\end{tabular}


Table.3 Effect of plant growth of Colocasia esculenta (Density) on wastewater treatment through various parameters at $100 \%$ concentration of sewage

\begin{tabular}{|l|l|l|l|l|l|l|l|}
\hline S.N. & Parameters & $\mathbf{0}$ Day & $\mathbf{1}^{\text {st }}$ Day & $\mathbf{2}^{\text {nd }}$ Day & $\mathbf{3}^{\text {rd }}$ Day & $\mathbf{4}^{\text {th }}$ Day & $\mathbf{5}^{\text {th }}$ Day \\
\hline 1. & $\begin{array}{l}\text { Plant Growth } \\
(\text { Numbers })\end{array}$ & 40 & 41 & 46 & 54 & 63 & 69 \\
\hline 2. & $\mathrm{pH}$ & 6.79 & 7.12 & 7.23 & 7.61 & 7.82 & 7.91 \\
\hline 3. & $\mathrm{EC}(\mu \mathrm{Moh} / \mathrm{Cm})$ & 2.63 & 2.17 & 1.72 & 1.02 & 0.98 & 0.99 \\
\hline 4. & $\mathrm{TSS}(\mathrm{mg} / \mathrm{L})$ & 402 & 339 & 201 & 115 & 103 & 111 \\
\hline 5. & $\mathrm{TDS}(\mathrm{mg} / \mathrm{L})$ & 1360 & 1043 & 726 & 502 & 471 & 481 \\
\hline 6. & $\mathrm{TS}(\mathrm{mg} / \mathrm{L})$ & 1762 & 1382 & 927 & 617 & 574 & 579 \\
\hline 7. & $\mathrm{BOD}(\mathrm{mg} / \mathrm{L})$ & 42.0 & 36.04 & 21.05 & 9.86 & 3.82 & 4.2 \\
\hline 8. & $\mathrm{COD}(\mathrm{mg} / \mathrm{L})$ & 121 & 93.7 & 37.9 & 24.3 & 11.86 & 12.87 \\
\hline 9. & $\mathrm{NO}_{3}(\mathrm{mg} / \mathrm{L})$ & 26.8 & 19.2 & 13.2 & 9.5 & 4.3 & 4.7 \\
\hline 10. & $\mathrm{PO}_{4}(\mathrm{mg} / \mathrm{L})$ & 24.60 & 18.81 & 12.46 & 8.67 & 2.92 & 3.2 \\
\hline 11. & $\mathrm{SO}_{4}(\mathrm{mg} / \mathrm{L})$ & 109.0 & 84.0 & 59.0 & 31.0 & 23.0 & 24.2 \\
\hline
\end{tabular}

Table.4 Effect of plant growth of Typha latifolia (Density) on wastewater treatment through various parameters at $100 \%$ concentration of sewage

\begin{tabular}{|l|l|l|l|l|l|l|l|}
\hline S. N. & Parameters & $\mathbf{0}$ Day & $\mathbf{1}^{\text {st }}$ Day & $\mathbf{2}^{\text {nd }}$ Day & $\mathbf{3}^{\text {rd }}$ Day & $\mathbf{4}^{\text {th }}$ Day & $\mathbf{5}^{\text {th }}$ Day \\
\hline 1. & Plant Growth (Numbers) & 40 & 47 & 59 & 68 & 77 & 84 \\
\hline 2. & $\mathrm{pH}$ & 6.62 & 7.59 & 7.52 & 7.49 & 7.48 & 7.82 \\
\hline 3. & EC $(\mu \mathrm{mohs} / \mathrm{cm})$ & 2.63 & 2.20 & 1.17 & 0.98 & 0.92 & 1.01 \\
\hline 4. & TSS $(\mathrm{mg} / \mathrm{L})$ & 335 & 297 & 210 & 134 & 96 & 102 \\
\hline 5. & TDS $(\mathrm{mg} / \mathrm{L})$ & 1631 & 1371 & 1064 & 752 & 548 & 551 \\
\hline 6. & $\mathrm{TS}(\mathrm{mg} / \mathrm{L})$ & 1966 & 1668 & 1274 & 886 & 644 & 653 \\
\hline 7. & $\mathrm{BOD}(\mathrm{mg} / \mathrm{L})$ & 46.0 & 32.0 & 21.4 & 14.7 & 4.26 & 4.39 \\
\hline 8. & $\mathrm{COD}(\mathrm{mg} / \mathrm{L})$ & 111.0 & 76.2 & 26.8 & 12.4 & 9.2 & 11.2 \\
\hline 9. & $\mathrm{NO}_{3}(\mathrm{mg} / \mathrm{L})$ & 21.0 & 17.6 & 13.1 & 9.2 & 3.0 & 3.6 \\
\hline 10. & $\mathrm{PO}_{4}(\mathrm{mg} / \mathrm{L})$ & 17.0 & 12.5 & 9.3 & 6.2 & 1.82 & 2.9 \\
\hline 11. & $\mathrm{SO}_{4}(\mathrm{mg} / \mathrm{L})$ & 96.0 & 88.0 & 67.0 & 58.0 & 46.0 & 54.0 \\
\hline
\end{tabular}

Table.5 Effect of plant growth of Pennisetum purpureium (Density) on wastewater treatment through various parameters at $100 \%$ concentration of sewage

\begin{tabular}{|l|l|l|l|l|l|l|l|}
\hline S. N. & Parameters & $\mathbf{0 ~ D a y ~}$ & $\mathbf{1}^{\text {st }}$ Day & $\mathbf{2}^{\text {nd }}$ Day & $\mathbf{3}^{\text {rd }}$ Day & $\mathbf{4}^{\text {th }}$ Day & $\mathbf{5}^{\text {th }}$ Day \\
\hline 1. & Plant Growth (Numbers) & 40 & 47 & 53 & 64 & 72 & 86 \\
\hline 2. & $\mathrm{pH}$ & 6.72 & 7.80 & 7.61 & 7.56 & 7.42 & 7.38 \\
\hline 3. & $\mathrm{EC}(\mu \mathrm{mohs} / \mathrm{cm})$ & 2.56 & 1.10 & 0.89 & 0.88 & 0.78 & 0.83 \\
\hline 4. & $\mathrm{TSS}(\mathrm{mg} / \mathrm{L})$ & 394 & 210 & 144 & 97 & 88 & 93 \\
\hline 5. & $\mathrm{TDS}(\mathrm{mg} / \mathrm{L})$ & 1364 & 1038 & 920 & 524 & 451 & 458 \\
\hline 6. & $\mathrm{TS}(\mathrm{mg} / \mathrm{L})$ & 1758 & 1248 & 1064 & 621 & 539 & 551 \\
\hline 7. & $\mathrm{BOD}(\mathrm{mg} / \mathrm{L})$ & 51.44 & 36.00 & 12.00 & 7.08 & 3.38 & 3.42 \\
\hline 8. & $\mathrm{COD}(\mathrm{mg} / \mathrm{L})$ & 118 & 90.16 & 29.80 & 18.20 & 8.07 & 8.09 \\
\hline 9. & $\mathrm{NO}_{3}(\mathrm{mg} / \mathrm{L})$ & 26.8 & 18.60 & 14.40 & 8.22 & 2.09 & 3.03 \\
\hline 10. & $\mathrm{PO}_{4}(\mathrm{mg} / \mathrm{L})$ & 21.60 & 16.40 & 11.58 & 6.50 & 2.32 & 2.40 \\
\hline 11. & $\mathrm{SO}_{4}(\mathrm{mg} / \mathrm{L})$ & 107 & 89.0 & 62.0 & 29.0 & 18.20 & 18.42 \\
\hline
\end{tabular}


Table.6 Effect of plant growth of Eichhornia crassipes (Density) on wastewater treatment through various parameters at $100 \%$ concentration of sewage

\begin{tabular}{|l|l|l|l|l|l|l|l|}
\hline S. N. & Parameters & $\mathbf{0 ~ D a y}$ & $\mathbf{1}^{\text {st }}$ Day & $\mathbf{2}^{\text {nd }}$ Day & $\mathbf{3}^{\text {rd }}$ Day & $\mathbf{4}^{\text {th }}$ Day & $\mathbf{5}^{\text {th }}$ Day \\
\hline 1. & Plant Growth (Numbers) & 40 & 49 & 57 & 66 & 78 & 91 \\
\hline 2. & $\mathrm{pH}$ & 6.81 & 7.97 & 7.86 & 7.57 & 7.42 & 7.34 \\
\hline 3. & $\mathrm{EC}(\mu \mathrm{mohs} / \mathrm{cm})$ & 0.6 & 1.3 & 1.9 & 2.2 & 3.2 & 3.7 \\
\hline 4. & $\mathrm{TSS}(\mathrm{mg} / \mathrm{L})$ & 1920 & 1773 & 1066 & 838 & 595 & 607 \\
\hline 5. & TDS $(\mathrm{mg} / \mathrm{L})$ & 1240 & 1772 & 768 & 712 & 545 & 547 \\
\hline 6. & TS $(\mathrm{mg} / \mathrm{L})$ & 3160 & 2945 & 1834 & 1550 & 1140 & 1154 \\
\hline 7. & $\mathrm{BOD}(\mathrm{mg} / \mathrm{L})$ & 230 & 220 & 200 & 165 & 118 & 120 \\
\hline 8. & $\mathrm{COD}(\mathrm{mg} / \mathrm{L})$ & 315 & 290 & 192 & 172 & 156 & 162 \\
\hline 9. & $\mathrm{NO}(\mathrm{mg} / \mathrm{L})$ & 9.2 & 8.0 & 6.0 & 5.1 & 1.23 & 3.0 \\
\hline 10. & $\mathrm{Chlorides} \mathrm{mg/L)}$ & 44.2 & 28.40 & 26.98 & 24.14 & 22.10 & 23.10 \\
\hline
\end{tabular}

Fig.1 Growth of Cana indica

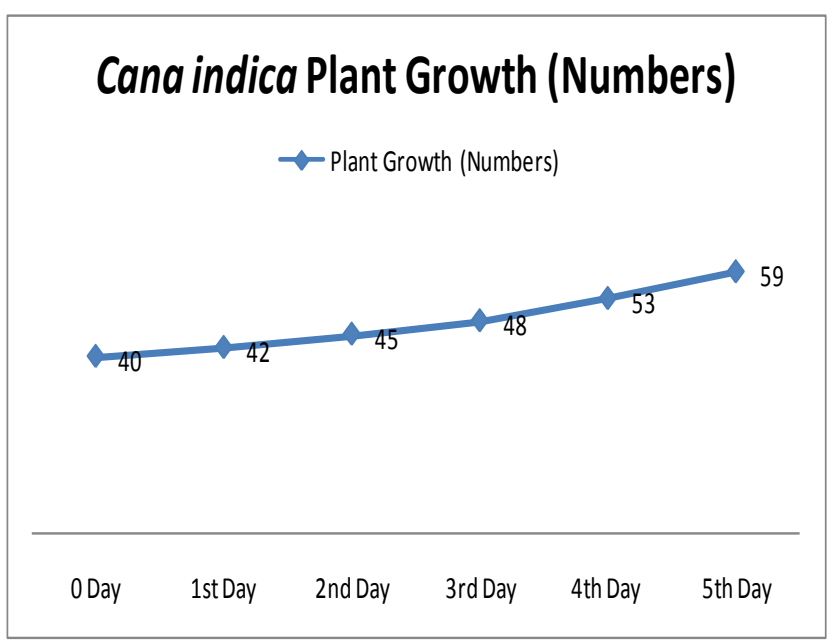

Fig.1 Growth of Panicum maximum

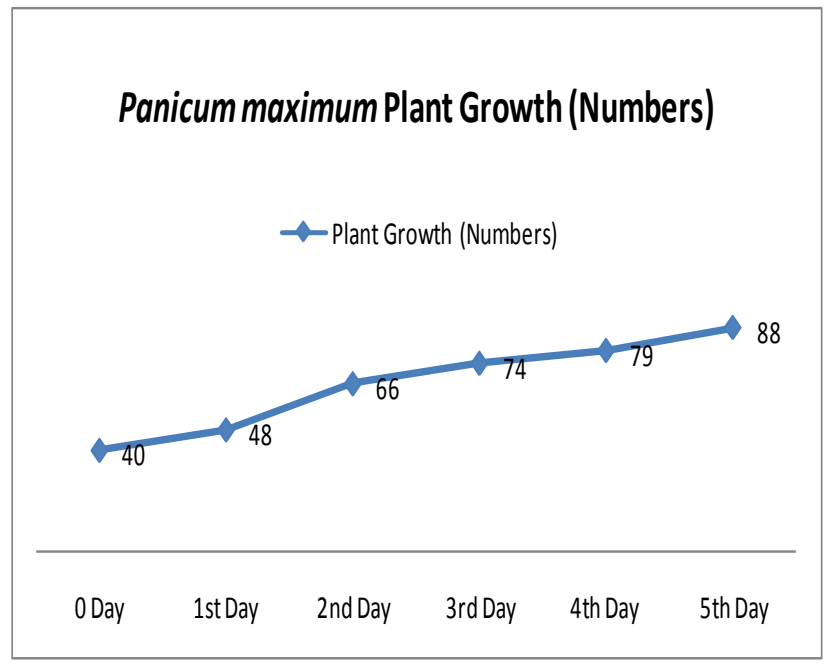


Fig.3 Growth of Colocasia esculenta

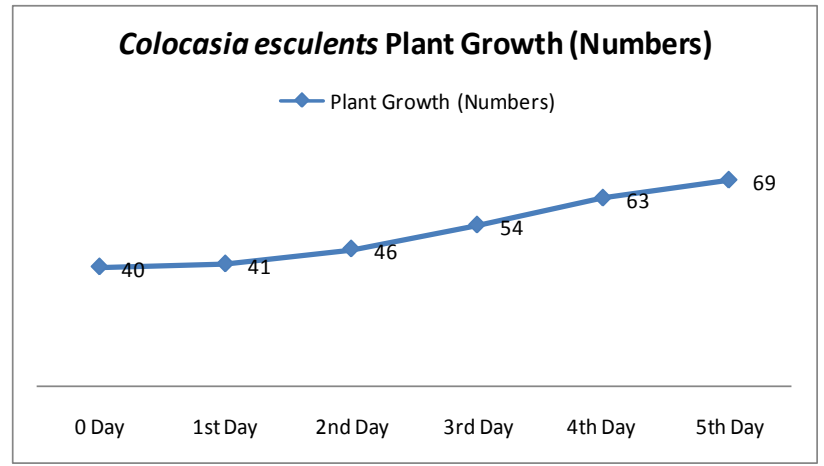

Fig.4 Growth of Typha latifolia

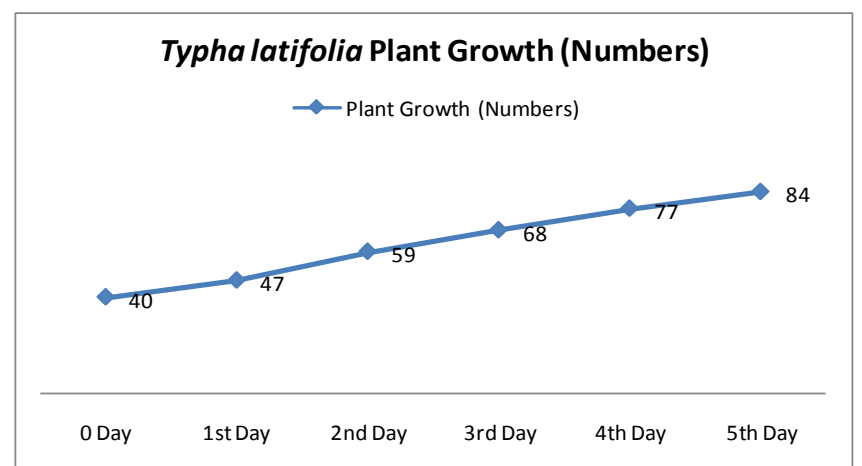

Fig.5 Growth of Pennisetum purpureium

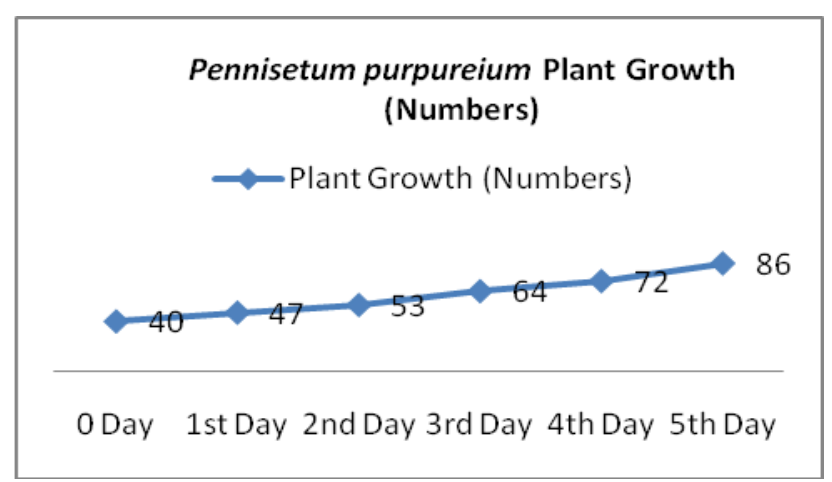

Fig.6 Growth of Eichhornia crassipes

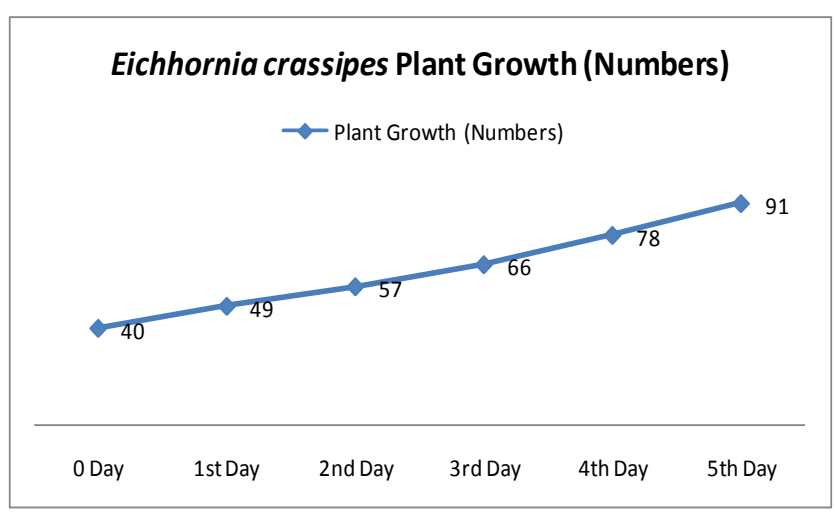


Typha latifolia shows that, plant biomass or number of Typha was increase 40 numbers at 0 day, 47 at $1^{\text {st }}$ day, 59 at $2^{\text {nd }}$ day, 68 at $3^{\text {rd }}$ day, 77 at $4^{\text {th }}$ day and 84 numbers at $5^{\text {th }}$ day respectively (Fig. 4). The $\mathrm{pH}$ values were changed by 6.62 at 0 day to 7.82 at $5^{\text {th }}$ day. BOD $(\mathrm{mg} / \mathrm{L})$ was reduced by 46.0 at 0 day to 4.26 at $4^{\text {th }}$ day. COD $(\mathrm{mg} / \mathrm{L})$ was reduced by 111 at 0 day to 9.2 at $4^{\text {th }}$ day respectively. Study showed the maximum pollutants removed at $4^{\text {th }}$ day (Table 4 ).

Pennisetum purpureium shows that, plant biomass or number of Pennisetum was increase 40 numbers at 0 day, 47 at $1^{\text {st }}$ day, 53 at $2^{\text {nd }}$ day, 64 at $3^{\text {rd }}$ day, 72 at $4^{\text {th }}$ day and 86 numbers at $5^{\text {th }}$ day respectively (Fig. 5). The $\mathrm{pH}$ values were changed by 6.72 at 0 day to 7.38 at $5^{\text {th }}$ day. BOD $(\mathrm{mg} / \mathrm{L})$ was reduced by 51.44 at 0 day to 3.38 at $4^{\text {th }}$ day. $\mathrm{COD}(\mathrm{mg} / \mathrm{L})$ was reduced by 118 at 0 day to 8.07 at $4^{\text {th }}$ day respectively. Study showed the maximum pollutants removed at $4^{\text {th }}$ day (Table 5).

Eichhornia crassipes shows that, plant biomass or number of Eichhornia was increase 40 numbers at 0 day, 49 at $1^{\text {st }}$ day, 57 at $2^{\text {nd }}$ day, 66 at $3^{\text {rd }}$ day, 78 at $4^{\text {th }}$ day and 91 numbers at $5^{\text {th }}$ day respectively (Fig. 6). The $\mathrm{pH}$ values were changed by 6.81 at 0 day to 7.34 at $5^{\text {th }}$ day. DO $(\mathrm{mg} / \mathrm{L})$ was increased by 0.6 at 0 day to 3.7 at $5^{\text {th }}$ day. BOD $(\mathrm{mg} / \mathrm{L})$ was reduced by 230 at 0 day to 118 at $4^{\text {th }}$ day. COD $(\mathrm{mg} / \mathrm{L})$ was reduced by 315 at 0 day to 156 at $4^{\text {th }}$ day respectively. Study showed the maximum pollutants removed at $4^{\text {th }}$ day, but the plant growth was increases continuously and plant bed observed the greenery, leafier and seems flowering (Table 6).

In conclusion, this overall study the Pennisetum purpureium of emergent and Eichhornia crassipes of free floating macrophytes removed greatest and maximum organic, inorganic pollutants from the sewage. All plant beds observed greenery, leafier and seem flowering due to this most of the faunal species was attracted towards the experimental setup of pilot plant.

\section{References}

Abdel Razik, A., Zidan., Mahmoud, M., ElGamal, Ahmed Ali Rashed, Mohamed Ahmed Abd El-Hady Eid. 2015. Wastewater treatment in horizontal subsurface flow constructedwetlands using different media (setup stage). Water Sci., 29: 26-35.

Abeer, M., Hegazya., Azza, H., ElSalakawy, Mohamed, M., Shaban, Mohamed, M., Yehia, Mohamed, S., AbuSalama. 2013. Eco-friendly management of enteroviruses in wastewater. Water Sci., 27: 19-29.

Baltic Deal (Internet). 2011. (cited April 2013). Agri-environmental Measures in the Baltic Sea Region - Advisory Services, Legislation and Best Practices, Available from: http://www.balticdeal.eu/documents/ agri-environmental-measures-in-thebaltic-sea-region-advisory-serviceslegislation-best-practices

Dhulap. V.P., Patil, S.S. 2014. Removal of Pollutants from Sewage through Constructed Wetland using Pennisetum purpureium. Int. European Acad. Res. J., Vol. II, Issue (1).

Dhulap, V.P., Ghorade, I.B., Patil, S.S. 2014. Seasonal study and its impact on sewage treatment in the angular horizontal subsurface flow constructed wetland using aquatic macrophytes. Int. J. Res. Engineering \& Technol., (IMPACT: IJRET)_ISSN (E): 2321-8843; ISSN 
(P): 2347-4599. Vol. 2, Issue - 213224.

Heers, M. 2006. Constructed wetlands under different geographic conditions: Evaluation of the suitability and criteria for the choice of plants including productive species. Master thesis, Faculty of Life Sciences, Hamburg University of Applied Sciences, Germany.

Kadlec, R.H., Wallace, S.D. 2008. Treatment Wetlands, 2nd ed. CRC Press, Boca Raton, FL, USA.

Kamau, C. 2009. Constructed wetlands: potential for their use in treatment of grey water in Kenya. M. Sc thesis, Christian-Albrechts University, Kiel, Germany.

Mayor, German, P., Sarana, J.R. 1950. The use of natural processes to remove pollutants in constructed wetlands has been extensively investigated and effectively applied in Western countries for decades. U. States, $T$.
Clean, Water Act.

Mohamed, A. 2004. Planung, Bau und Betrieb einer Pflanzenkläranlage in Syrien (Planning, construction and operation of a constructed wetland in Syria, in German). Ph.D thesis, University Flensburg, Germany.

Salomon, E., Sundberg, M. Internet. 2012. (Cited March 2013). Implementation and status of priority measures to reduce nitrogen and phospho-rus leakage - Summary of country reports, WP3. In: Baltic Compass Project Report, WP3. JTI., Available from:

http://www.balticcompass.org/PDF/ Reports/SummaryOfCountryReports. pdf

Wallace, S.D., Knight, R.L. 2006. Small Scale Constructed Wetland Treatment Systems. Feasibility, Design Criteria, and O\&M Requirements.Water Environ. Res. Foundation, Alexandria, VA, USA.

\section{How to cite this article:}

Dhulap, V.P., and Patil, S.S. 2016. Impact of Plant Density on the Sewage Treatment through selected Aquatic Macrophytes Using Angular Horizontal Subsurface Flow Constructed Wetland. Int.J.Curr.Microbiol.App.Sci. 5(6): 97-104. doi: http://dx.doi.org/10.20546/ijcmas.2016.506.012 